\title{
THE EFFECT OF COALESCENCE IN CERTAIN COLLISION PROCESSES*
}

\section{BY \%. ALEXANDER MFLZAK (.MeGill C'nirersit!)}

This note is concerned with the variable size-distributions of atmospheric and colloidal particles. The following physical model is adopted: a large number of particles are randomly distributed in space; these particles are in random motion due to e.g. Brownian effect or turbulence; the system of particles is given by the particle-mass distribution. The distribution varies as a result of collision processes in which the randomly moving particles meet and coalesce. The particles do not break up and do not enter or leave the system under consideration. It is assumed that the total number of particles is large enough to justify the use of

(a) the mass density function $f(x, t) ; f(x, t) d x$ being the average number per unit volume of particles of mass (or volume) $x$ to $x+d x$ at time $t$,

(b) the coalesience function $\phi(u, v)$, where

$$
. V(u, v, t)=f(u, t) f(v, t) \phi(u, v) d u d v d t,
$$

$N(u, r, t)$ being the average number, per unit volume, of collisions resulting in coalescence, between particles of mass $u$ to $u+d u$ and $v$ to $v+d v$ respectively, in the time interval $t$ to $t+d t$.

The function $\phi(u, v)$ takes care of such factors as: the collision cross-section, the mobility of the particles, the fraction of collisions resulting in coalescence and any shape factor affecting the collision. With these definitions, the condition for the conservation of mass becomes:

$$
\frac{\partial f(x, t)}{\partial t} d x d t=\frac{1}{2} \int_{u+v=x} N(u, v, t)-\int_{u=0}^{\infty} N(u, x, t) .
$$

This means that the increase of the number of particles of mass $x$ in time $d t$ is equal to half the number of coalescences between particles of masses $u$, and $x-u, 0 \leq u \leq x$, minus the number of coalescences between the particles of mass $x$ and any others. Substituting equation (1) in equation (2) gives the fundamental equation of the process:

$$
\frac{\partial f(x, t)}{\partial t}=\frac{1}{2} \int_{0}^{x} f(y, t) f(x-y, t) \phi(y, x-y) d y-f(x, t) \int_{0}^{\infty} f(y, t) \phi(x, y) d y .
$$

If $N(t)$ is the total number of particles (per unit volume) then:

$$
\begin{aligned}
N(t) & =\int_{0}^{\infty} f(x, t) d x, \\
\frac{d N(t)}{d t} & =-\frac{1}{2} \int_{0}^{\infty} \int_{0}^{\infty} f(x, t) f(y, t) \phi(x, y) d x d y .
\end{aligned}
$$

The initial condition will be taken as $f(x, 0)=g(x)$, a known function corresponding to a known initial distribution. If this initial distribution is nearly homogeneous, and if $\phi(x, y)$ does not oscillate rapidly, then for small $t$ equation (5) may be approximated by:

$$
\frac{d N}{d t}=-\frac{1}{2} \int_{n}^{\infty} \int_{n}^{\infty} f(x, t) f(y, t) c d x d y=-\frac{c N^{2}}{2}, \quad c=\text { const. }
$$

${ }^{*}$ Received June 23. 1952. The research reported upon has been sponsored by the Geophysics Research Division of the Air Force Cambridge Research Center under Contract AF 19(122)-217. 
This may be regarded as a theoretical foundation of an empirical coagulation law valid for some cases of Brownian motion. Whytlaw-Grey (1932) and Sinclair (1950) discuss the range and validity of equation (6) in more detail.

Of course, equation (6) holds exactly if $\phi(x, y) \equiv c$. In this case, one can solve the main equation (3) under general initial conditions. Letting $2 F(x, t)=c f(x, t)$, equation (3) becomes:

$$
\frac{\partial F(x, t)}{\partial t}=\int_{0}^{x} F(y, t) F(x-y, t) d y-2 F(x, t) \int_{0}^{\infty} F(y, t) d y .
$$

One introduces the Laplace Transforms:

$$
\psi(p, t)=\int_{0}^{\infty} e^{-p x} F(x, t) d x ; \quad \psi(p, 0)=\frac{c}{2} \int_{0}^{\infty} e^{-p x} g(x) d x .
$$

Then, using the standard properties of the Laplace Transforms, one obtairs from equattion $(\overline{\boldsymbol{}})$ the equation for $\psi(p, t)$ :

$$
\frac{\partial \psi(p, t)}{\partial t}=\psi^{2}(p, t)-2 \psi(p, t) \psi(0, t) ; \quad \psi(p, 0) \text { known }
$$

Its solution is:

$$
\psi(p, t)=\frac{1}{(N t+1)^{2}} \frac{\psi(p, 0)}{1-\frac{t}{N t+1} \psi(p, 0)} ; \quad N=\psi(0,0)=\frac{C N(0)}{2} .
$$

Now, inverting equation (8), one obtains the final solution:

$$
f(x, t)=\frac{2}{C(N t+1)^{2}} \frac{1}{2 \pi i} \int_{\gamma-i \infty}^{\gamma+i \infty} \frac{e^{p x} \psi(p, 0)}{1-\frac{t}{N t+1} \psi(p, 0)} d p ;
$$

$\gamma>0$ and sufficiently large.

(From equation (11), one may obtain as a particular case a solution of equation (3) given by Schumann (1940), valid for a specific initial distribution only.) The conditions under which the above use of the Laplace Transform is justified are not discussed here, but it is easy to see from the physical situation that they are satisfied.

A few particular results for initial distributions which may be of interest have been worked out. Two of them follow. then

$$
\text { If } f(x, 0)=g(x)=A \delta(x-1),
$$

$$
f(x, t)=\frac{2 N}{C(N t+1)^{2}} \sum_{i=0}^{\infty}\left(\frac{N t}{N t+1}\right)^{i} \delta[x-(j+1)] ; \quad N=\frac{C A}{2} .
$$

From equation (12) it follows, as is otherwise clear, that starting with single particles of equal size one will later have double, triple, $m$-tuple aggregates. The number of $m$-tuples per unit volume at time $t$ is $\gamma(t) e^{-m \beta(t)}$. then

If $g(x)=f(x, 0)=A x^{n} e^{-\alpha x}, n$ integer,

where

$$
f(x, t)=\frac{2 e^{-\alpha x} L}{c t(N t+1)(n+1)} \sum_{i=1}^{n+1} \zeta^{i} \exp \left(\zeta^{i} L x\right),
$$

$$
2 N=\frac{C A n !}{\alpha^{n+1}}, \quad L=\left[\frac{c n ! t A}{2(N t+1)}\right]^{1 / n+1}, \quad \zeta=e^{2 \pi i / n+1} .
$$


Equation (13) shows on closer analysis that as $n$ increases the oscillatory terms become more and more dominant, giving progressively sharper and higher periodic peaks in $f(: t)$.

Equation (3) was also solved under different assumptions for $\phi(x, y)$ viz. $\phi(x, y)=$ $\boldsymbol{c}(x+y)$ and $\phi(x, y)=c x y$. The solutions, except for some special initial conditions, are involved, though quite straightforward. They will not be reproduced here, but may be obtained by transforming equation (3) by means of equations (4) and (5), introducing Laplace Transforms and solving the resulting partial differential equations.

With physically more realistic assumptions for $\phi(x, y)$, e.g. $\phi(x, y)=c\left(x^{1 / 3}+y^{1 / 3}\right)^{2}$ (capture cross-section for two spheres), equation (3) becomes so involved that it has to be treated numerically.

The following meteorological problem provides an illustration of the theory dereloped above. Marshall and Palmer (1948) found a simple expression for the distribution of the raindrop sizes. Their exponential relation,

$$
N_{D}=N_{0} C^{-1 D} \text {, }
$$

where $N_{D} d D$ is the number of drops in the diameter interval $D-D+d D$ in unit value of space,

$N_{0}$ is a universal constant,

$\Lambda$ is a rain-intensity parameter,

fits the observed data of Laws and Parsons (1943) very well except at small diameters. This deviation at small diameters may be explained by assuming the distribution given by equation (14) subject to a collision process with the above form of $\phi(x, y)$. The results are shown in figure 1 . An attempt was also made to explain theoretically the whole dis-

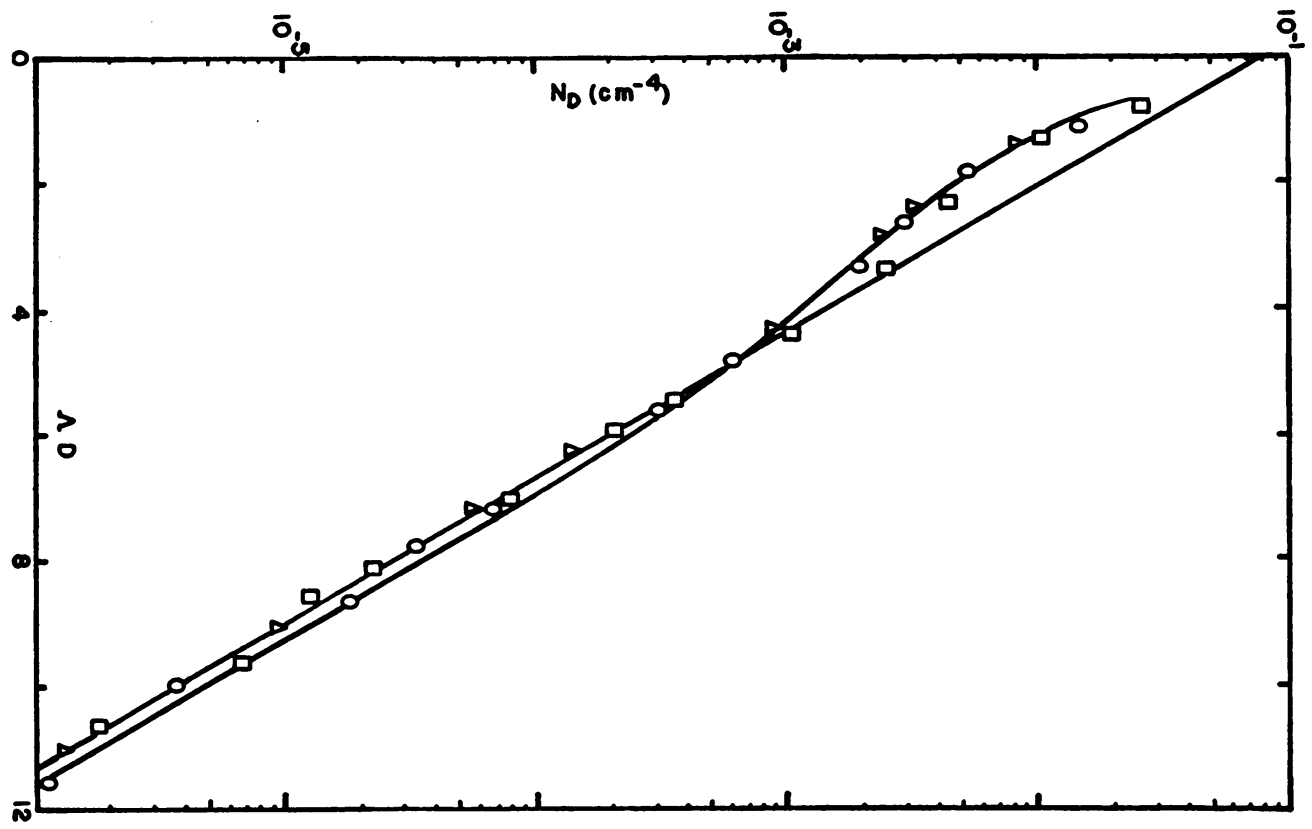

Fig. 1. The marked points represent the experimental data of Laws and Parsons, the straight line is obtained from the Marshall-Palmer relation, the curved line represents the calculated distribution. $N_{D}, \Lambda, D$ are explained under Eq. (14) in the text. 
tribution given by (14), but the results are not yet satisfactory. This means probably that the form of $\phi(x, y)$, chosen above, does not describe the physical situation sufficiently closely.

The author is indebted to Professor J. S. Marshall, of McGill University, for suggesting the above problem, and to Dr. Walter Hitschfeld, of McGill University, for helpful suggestions concerning the physical model used.

\section{REFERENCIS}

J. O. Laws and I). A. Parsons, The relation of raindrop-size to intensit!y, Trans. Amer. Geophys. Union, 24, part II, $452(19+3)$.

J. S. Marshall and W. MCK. Palmer, The distribution of raindrops with size, J. Meteor, 5, 4, 165 (1948).

T. E. W. Schumann, Theoretical aspects of the size distribution of $\log$ particles, Q. J. Roy. Met. Soc., 66 (1940).

D. Sinclair, Handbook on Aerosols. Chapter 5, Atomic Energy Commission, Washington (1950).

Whytlaw-Grey, Smoke. L. Arnold and Co., London (1932).

\section{VARIATION OF COEFFICIENTS OF SIMULTANEOUS LINEAR EQUATIONS ${ }^{1}$}

By JOHN E. BROCK (Midwest Piping Supply Co., Inc.)

1. Introduction. In dealing with sets of simultaneous linear equations it sometimes becomes necessary to modify one or more of the coefficients (to correct errors or for other reasons) after the solution has been obtained. Methods of obtaining the corresponding modifications in the solution with a minimum amount of computation have been treated by B. L. Weiner ${ }^{2}$, and in a discussion of Weiner's paper (which bears the same title as the present paper), I. F. Morrison ${ }^{3}$ indicates a matrix formulation of the analysis. In the present paper, we develop three particular prodedures each of which appears to be quite simple and quite useful.

Matrices are used throughout in these developments. Both matrices and scalars will be denoted by ordinary italic letters. Letters with subscripts will denote matrix elements (scalars) and other scalars will be easily distinguished from matrices. A symbol denoting a matrix or matrix product when enclosed within parentheses and followed by subscripts will denote the appropriate element of the matrix, thus $(a b)_{i j}=c_{i j}$ where $a b=c$. Superscripts will be used both to denote exponents and to serve as distinguishing indices; there should be no difficulty in distinguishing between these usages.

It is presumed that we know the set of inverse coefficients for the system, that is, the matrix which is inverse to (reciprocal to) the original coefficient matrix. This is not a severe demand since the reciprocal matrix is frequently already known or can be computed without much trouble making use of calculations already performed in obtaining the original solution.

So that the operations will not be obscured by voluminous calculations, the order, $n$, of the systems in the examples chosen for illustrative purposes is small. However, it is obvious that the advantage afforded by the procedures described here increases as $n$ increases.

${ }^{1}$ Received June 15, 1952.

${ }^{2}$ B. L. Weiner, Variation of coefficients of simultaneous linear equations, Trans. ASCE, 113, 1349 (1948) (Paper No. 2358).

'I. F. Morrison, ibid., p. 1379. 